\title{
Unmanned parking method based on cloud platform
}

\author{
Liang $\mathrm{Yu}^{1}$, Luo Jie ${ }^{2}$, Luo Haoru ${ }^{3}$, Liu Sijia ${ }^{4}$ and Yang $\mathrm{Xu}^{*}$ \\ ${ }^{1}$ School of Automation, Wuhan University of Technology, 122 Luoshi Road, Hongshan District, Wuhan, China \\ ${ }^{2}$ School of Automation, Wuhan University of Technology, 122 Luoshi Road, Hongshan District, Wuhan, China \\ ${ }^{3}$ School of Automation, Wuhan University of Technology, 122 Luoshi Road, Hongshan District, Wuhan, China \\ ${ }^{4}$ School of Automation, Wuhan University of Technology, 122 Luoshi Road, Hongshan District, Wuhan, China \\ ${ }^{*}$ School of Automation, Wuhan University of Technology, 122 Luoshi Road, Hongshan District, Wuhan, China
}

\begin{abstract}
With the intelligent development of automobile driving, automatic parking technology is widely concerned by relevant technicians at home and abroad. Automatic parking technology is a method that can realize automatic parking in and out of parking Spaces without manual intervention. At present, automatic parking system has been equipped and used in many models, but there are many problems such as low parking accuracy and unable to effectively identify irregular parking Spaces.In order to solve the above problems, this article is based on a cloud platform developed a driverless car parking method, through the cloud server, GPS (Global Positioning System) and inertial navigation.It can according to the parking information and real-time Positioning planning adjustment path of the vehicle, and controls the complete vehicle posture adjustment, so as to improve the accuracy of parking and parking intelligent identification accuracy and ensure the safety and efficiency in the process of path planning. The effectiveness of the method is verified by simulation experiments with MATLAB software, which can be demonstrated by data.
\end{abstract}

\section{Introduction}

With the rapid development of science and technology and social economy, the scale of China's automobile industry continues to expand. People's increasing demand for the use of cars makes the number of cars in China continue to rise in recent years. In addition to causing the road traffic burden and increasing the probability of traffic accidents, the problem of difficult urban parking has become particularly prominent ${ }^{[1]}$. The increasing number of cars and the city's inadequate parking infrastructure are increasingly unbalanced, resulting in a shortage of parking Spaces. In order to improve the utilization of space resources, the size of the parking lot will be very limited. In the face of narrow parking Spaces, many drivers are not able to grasp the parking distance and steering timing well. Meanwhile, the influence of the blind area behind the vehicle on the reversing vision is easy to lead to the wrong judgment of the overall perception of the vehicle, thus leading to certain parking accidents ${ }^{[2]}$.

Using the parking assistance system can improve the safety and efficiency of parking to a certain extent. The system helps the driver to reduce the parking time, but also can avoid the misjudgment in the field of vision, so as to reduce the difficulty of parking. At present, the parking assistance system is committed to providing drivers with a broader parking vision and active detection of surrounding obstacles, so as to improve the safety and work efficiency of parking [3]. Automatic parking technology is a kind of intelligent driving assistance technology, which can replace the driver to accurately complete the operation of vehicle storage, realize the vehicle to find parking space, identify parking space, vehicle safety parking and other functions, greatly shortening the parking time, to avoid the unnecessary risk of parking collision. At the same time, the parking standard guided by automatic parking will surely save space resources and improve the chaotic parking situation in the city. The development of automatic parking technology is closely related to the development of intelligent vehicles, which promotes the construction of intelligent transportation system to a certain extent and has a broad market prospect.

\section{Theory}

The automatic parking system needs to meet certain speed conditions when it is enabled. Generally, it can realize two or three of the functional parking modes of vertical parking, horizontal parking and oblique parking, and there are minimum requirements for both the length and width of parking Spaces in the identification of parking Spaces ${ }^{[4]}$. At present, the interactive auxiliary system that can realize automatic parking is mostly used.

When the driver drives the car into the parking lot, he needs to park the car in a position parallel to the parking space and the distance to the parking space meets certain requirements. The central control screen or instrument button is operated to open the parking function. The parking system uses visual or ultrasonic sensors to detect space and line parking Spaces. When an effective parking

\footnotetext{
* Corresponding author: yx_auto@whut.edu.cn
} 
space is detected, the driver is prompted to select the parking space and start parking. The parking system plans the parking path and controls the parking space. However, in the process of automatic parking, visual and ultrasonic sensors are first used to detect parking Spaces. However, light and weather often affect the detection results of visual and ultrasonic sensors ${ }^{[5]}$, thus affecting the effect of automatic parking. In addition, the driver should first drive the car to an appropriate position, so that the vehicle posture before parking will have a great impact on the effect of automatic parking. If the deviation of the vehicle posture is too large, it is likely to lead to parking failure.

In order to solve the above technical problems, this paper proposes an unmanned parking method based on the cloud platform, which can automatically plan and realtime update the parking track and control the vertical and horizontal movement of vehicles according to the target parking space coordinate information and real-time vehicle positioning information transmitted by the cloud. To adjust the position and posture of parked vehicles before parking can improve the accuracy of parking and reduce the possibility of parking failure, so that the vehicles to be parked can park into the parking space more accurately and faster. This automatic parking method needs to adjust the posture and trajectory planning of the parked vehicles on the basis of obtaining the target parking space information, and finally control the parked vehicles to park into the target parking space according to the parking trajectory. The method flow is shown in Figure 1.

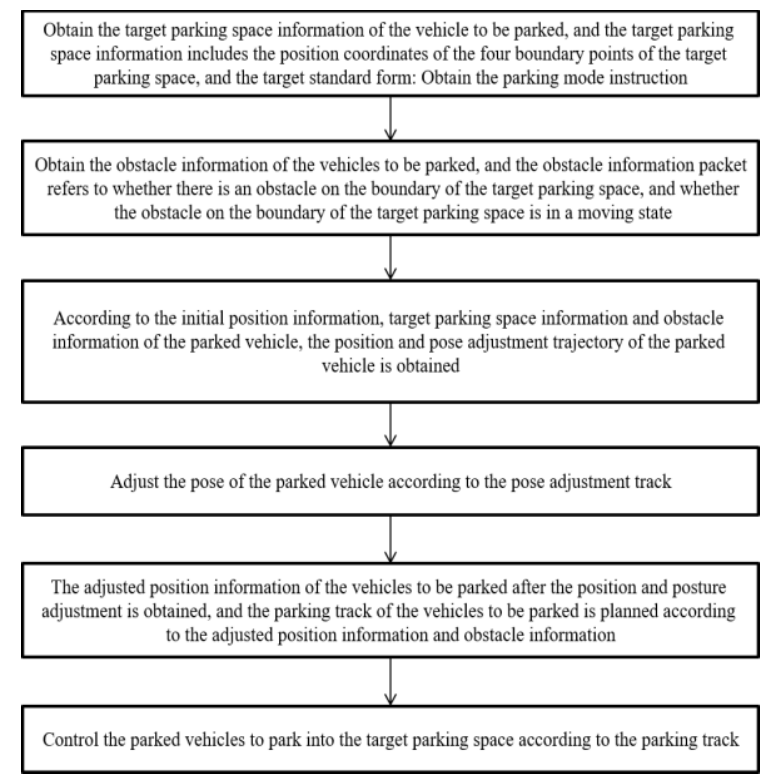

Figure 1. The flow diagram of automatic parking method

\subsection{Acquisition of target parking space information}

Car before entering the park environment, first of all need to get the target parking information, the driver through for cars parked parking requests to the cloud service platform control system, including the initial position information of cars parked, again through the cloud service platform for the goal of cars parked car, cloud service platform based on receiving request for parking for cars parked goal set parking, and will target parking information transmission and the way of anchored into the instruction to control system.

\subsection{Acquisition of pose trajectory}

The position and pose adjustment trajectory of the vehicle to be parked can be obtained according to the initial position information, target parking space information and obstacle information.

Firstly, the obstacle information is processed, and the position information of the obstacle is obtained by each sensor, and the coordinates of the obstacle are determined by the perceptual fusion algorithm. According to the initial position information and the target parking space information, the initial position coordinates and the target parking space coordinates of the vehicle to be parked are calculated respectively. Transform the initial position coordinates of the parked vehicle and the target parking space coordinates to get the initial position coordinates and the target parking space coordinates of the parked vehicle in the $\mathrm{P}$ coordinate system. The coordinate transformation formula is as follows:

$$
\left[\begin{array}{ll}
x^{\prime} & y^{\prime}
\end{array}\right]=\left[\begin{array}{ll}
x-d_{x} & y-d_{y}
\end{array}\right]\left[\begin{array}{cc}
\cos \theta & -\sin \theta \\
\sin \theta & \cos \theta
\end{array}\right]
$$

Wherein, $\mathrm{x}$ 'and $\mathrm{y}^{\text {' }}$ are the converted coordinates of $\mathrm{P}$ system; $\mathrm{x}$ and $\mathrm{y}$ are the coordinates in the geodetic coordinate system; $\theta$ is the rotation Angle of coordinates; $d_{x}, d_{y}$ are the coordinates of the origin of coordinate system in the geodetic coordinate system.

The path points under p-system need to be calculated according to the starting point of the vehicle parking track, obstacles and the coordinate value of the vehicle under $p$ system. In the specific calculation, the abscissa of the path points is determined by linear interpolation of the abscissa of the vehicle under the p-system and the abscissa of the obstacle under the p-system, and the map is extended 15 meters forward after the interpolation is completed. The ordinate of the path point is equal to the coordinate of the starting point of the parking in the p-system, and the constant value remains unchanged. Thus, the pose adjustment trajectory of the parked vehicle in the $\mathrm{P}$ coordinate system is obtained. Then, coordinate transformation is carried out to obtain the pose adjustment trajectory of the parked vehicle in the geodetic coordinate system. The formula of coordinate transformation is as follows:

$$
\left[\begin{array}{ll}
x^{\prime} & y^{\prime}
\end{array}\right]=\left[\begin{array}{ll}
x & y
\end{array}\right]\left[\begin{array}{cc}
\cos \theta & \sin \theta \\
-\sin \theta & \cos \theta
\end{array}\right]+\left[\begin{array}{ll}
d_{x} & d_{y}
\end{array}\right]
$$

Wherein, $\mathrm{x}$ 'and $\mathrm{y}^{\prime}$ are the converted coordinates of $\mathrm{P}$ system; $\mathrm{x}$ and $\mathrm{y}$ are the coordinates in the geodetic coordinate system; $\theta$ is the rotation Angle of coordinates; 
$d_{x}, d_{y}$ are the coordinates of the origin of coordinate system in the geodetic coordinate system.

\subsection{Parking trajectory planning after position and pose adjustment}

After the position and posture of the vehicle to be parked is adjusted, its adjusted position information needs to be obtained, and the parking track of the vehicle to be parked should be re-planned according to the adjusted position information and obstacle information.

Firstly, coordinate transformation of the adjusted position coordinates of the parked vehicles is needed to obtain the adjusted position coordinates of the parked vehicles in the $\mathrm{P}$ coordinate system. Then the adjusted position coordinates of the parked vehicle in the $\mathrm{P}$ coordinate system and the coordinate of the target parking space are used to calculate the transverse distance between the parked vehicle and the target parking space in the $\mathrm{P}$ coordinate system. In the specific calculation, if there is no obstacle in the pose adjustment stage, the default value of transverse distance is adopted. If there is an obstacle in the pose adjustment stage, the transverse distance is the larger value between the default value and the $\mathrm{Y}$ coordinate value of the obstacle in the $\mathrm{p}$-system plus 0.5 .

Secondly, the width and depth of the target parking space should be determined, and the relative position of the vehicle to be parked and the target parking space should be obtained according to the width and depth of the target parking space. Through rotation and transformation, the coordinates of the vehicle target parking spot in the geodetic coordinate system are converted to the vehicle coordinate system, and the conversion formula is as follows:

$$
\left[\begin{array}{ll}
x^{\prime} & y^{\prime}
\end{array}\right]=\left[\begin{array}{ll}
x_{\text {spot }}-x_{\text {car }} & y_{\text {spot }}-y_{\text {car }}
\end{array}\right]\left[\begin{array}{cc}
\cos \theta & -\sin \theta \\
\sin \theta & \cos \theta
\end{array}\right]
$$

Where: $\mathrm{x}$ 'and $\mathrm{y}$ ' are the coordinates of the target parking spot in the coordinate system of the converted vehicle; $x_{\text {spot }}$ and $y_{\text {spot }}$ are the coordinates of the target parking spot in the geodetic coordinate system; $\theta$ is the rotation Angle of coordinates; $x_{c a r}$ and $y_{c a r}$ are the coordinates of the vehicle in the geodetic coordinate system.

Finally, the Angle between the center line of the target parking space and the $\mathrm{Y}$ axis of the $\mathrm{P}$ coordinate system is calculated, and the parking boundary constraint of the vehicle to be parked is obtained through the sensing technology.

According to the parameters, position information, target parking space information and obstacle information of the vehicle to be parked, the parking track is calculated to obtain the coordinates of the parking track of the vehicle to be parked in the $\mathrm{P}$ coordinate system. The coordinate relation diagram is shown in Figure 2. The calculation method of the coordinates of the parking track of the vehicle to be parked in the $\mathrm{P}$ coordinate system is as follows:

$$
\begin{gathered}
x_{o 1}=x_{1} \\
y_{o 1}=y_{1}+R \\
x_{2}=x_{o 1}+R^{*} \sin (a 1) \\
y_{2}=y_{o 1}-R^{*} \cos (a 1) \\
a 2=90-a 1 \\
x_{o 2}=x_{2}+R \cos (a 2) \\
y_{o 2}=y_{2}-R * \sin (a 2) \\
x_{3}=x_{o 2}-R=0
\end{gathered}
$$

Wherein, $\mathrm{P} 1$ is the forward starting turning point of the vehicle to be parked, $\mathrm{P} 2$ is the backward shifting point of the vehicle to be parked, P3 is the backward straight going point of the vehicle to be parked, and $\mathrm{P} 4$ is the parking end stopping point of the vehicle to be parked. $\mathrm{O} 1$ and $\mathrm{O} 2$ are the center points of the arcs taken by the parked vehicles in the two steering processes respectively; $\mathrm{R}$ is the minimum turning radius; $\mathrm{A} 1$ and $\mathrm{A} 2$ are the central angles corresponding to the two steering arcs respectively; $(x, y)$ are the coordinates of the above points in the re-p coordinate system respectively.

After obtaining the coordinate of the parking track of the parked vehicle in the $\mathrm{P}$ coordinate system, coordinate transformation is carried out to obtain the coordinate of the parking track of the parked vehicle in the geodetic coordinate system.
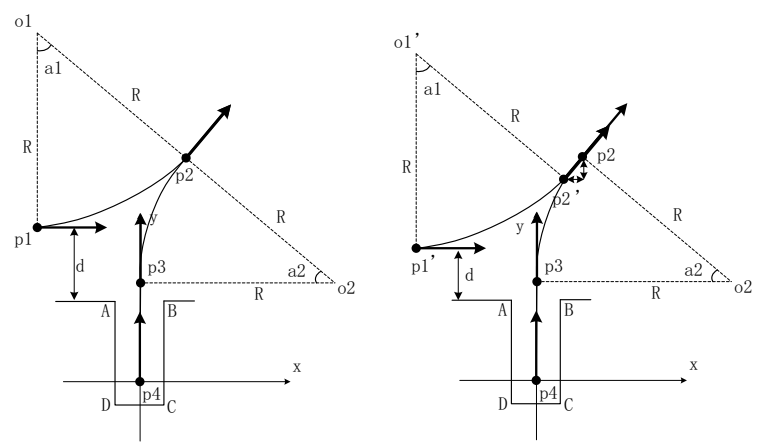

Figure 2. Coordinates diagram of parked vehicles

\subsection{Parking Lateral control and longitudinal control of the target parking space}

When controlling the parked vehicle to park to the target parking space, it needs to carry out lateral control and longitudinal control.

When conducting lateral control, the real-time position coordinates and preview distance of the vehicles to be parked need to be obtained first. The calculation formula of preview distance is as follows: 


$$
l d=c_{0} v^{2}+\mathrm{c}_{1} v+\mathrm{c}_{2}
$$

Whereinto, $c_{0}, c_{1}$ and $c_{2}$ are the coefficients to be calibrated.

Secondly, according to the real-time position and preview distance of the vehicle to be parked, the preview point in the parking track is determined by comparing whether the distance between the planned path point and the vehicle is equal to the preview distance. Through rotation and transformation, the coordinates of the preview point in the geodetic coordinate system are converted to the vehicle coordinate system, and the preview deviation of the preview point is calculated. The coordinate transformation formula is as follows:

$$
\left[\begin{array}{ll}
x^{\prime} & y^{\prime}
\end{array}\right]=\left[\begin{array}{ll}
x_{l p}-x_{c a r} & y_{l p}-y_{c a r}
\end{array}\right]\left[\begin{array}{cc}
\cos \theta & -\sin \theta \\
\sin \theta & \cos \theta
\end{array}\right]
$$

Wherein, $x$ 'and $y$ ' are the coordinates of the pre-view point in the converted vehicle coordinate system; $x_{l p}$ and $y_{l p}$ are the coordinates of the pre-view point in the geodetic coordinate system; $\theta$ is the rotation Angle of coordinates; $x_{c a r}$ and $y_{c a r}$ are the coordinates of the vehicle in the geodetic coordinate system; $y^{\prime}$ to the prime is the preview deviation

According to the obtained preview deviation, the wheel Angle of the vehicle to be parked is calculated by PID. The calculation formula of the wheel Angle is as follows:

Where: $\delta_{\text {wheel }}$ is the wheel turning Angle; "Err" is preview deviation; "Kp" is the proportional control coefficient; "Kd" is the differential control factor;

$$
\delta_{\text {wheel }}=\left(k_{p} * e r r+k_{d} * e r r\right)
$$

According to the wheel Angle lookup table, the requested steering wheel Angle can be obtained to complete the lateral control of parked vehicles.

When the longitudinal control is carried out, the realtime position of the vehicle to be parked should be obtained first, and the longitudinal control mode and gear of the vehicle to be parked should be determined according to the real-time position of the vehicle to be parked and the pre-stored control information of the control system.

Secondly, the speed control mode of the vehicle to be parked should be determined according to the actual path. When the distance from the vehicle to the shift point is greater than the set threshold value, the constant speed control mode is used. When the distance between the vehicle and the shift point is less than the set threshold, the fixed-point parking control mode is used. The calculation formula of the actual distance of the parked vehicle is as follows:

$$
\mathrm{S}=\int v
$$

Where: $\mathrm{S}$ is the actual distance; $\mathrm{V}$ is the speed.

Finally, the control system carries out longitudinal control to the parked vehicles according to the longitudinal control mode, gear and speed control mode.

\section{Experimental}

\subsection{Path planning simulation}

In this paper, "JAC Electric Vehicle" is used as theexperimental platform, and its parameters are shown in Table 1. According to the planning method, the simulation is carried out in "Matlab" to verify the feasibility of path planning.

Table 1. Experimental vehicle parameters (m)

\begin{tabular}{|c|c|c|c|c|c|}
\hline Project & $\begin{array}{c}\text { Front } \\
\text { suspen } \\
\text { sion } l_{f}\end{array}$ & $\begin{array}{c}\text { Rear } \\
\text { suspen } \\
\text { sion } l_{r}\end{array}$ & $\begin{array}{c}\text { Wheel } \\
\text { base } \\
l\end{array}$ & $\begin{array}{c}\text { Car } \\
\text { lengt } \\
\mathbf{h} L\end{array}$ & $\begin{array}{c}\text { Car } \\
\text { width } \\
\boldsymbol{W}\end{array}$ \\
\hline $\begin{array}{c}\text { Paramet } \\
\text { er }\end{array}$ & 0.800 & 0.800 & 2.490 & 4.135 & 1.750 \\
\hline
\end{tabular}

The path planning is shown in Figure 3. The minimum parking length is $6.73 \mathrm{~m}$, and the ratio of minimum parking length to vehicle length is 1.63 . Vorobieva et al. ${ }^{[6]}$ used a simulation vehicle with a length of $4.084 \mathrm{~m}$ and a minimum parking space of $6.8 \mathrm{~m}$ for a single parking, with a ratio of 1.67 .

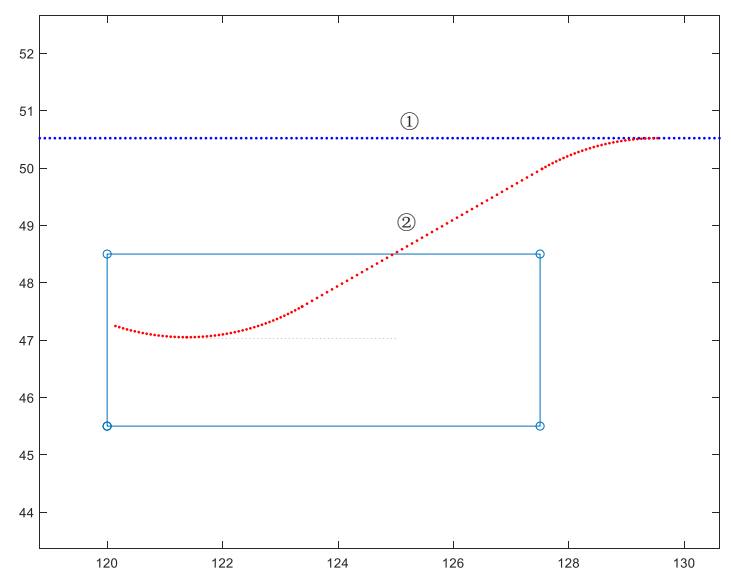

Figure 3. Path planning

We use a roundabout curve in the parking path planning, which ensures the continuous curvature of the parking process and reduces the length of the required parking space to a certain extent. The simulation results of parking path planning are shown in Figure 4. At the end coordinate of parking, the vehicle is parallel to the parking space and the path is smooth, which can complete the task of path planning. 


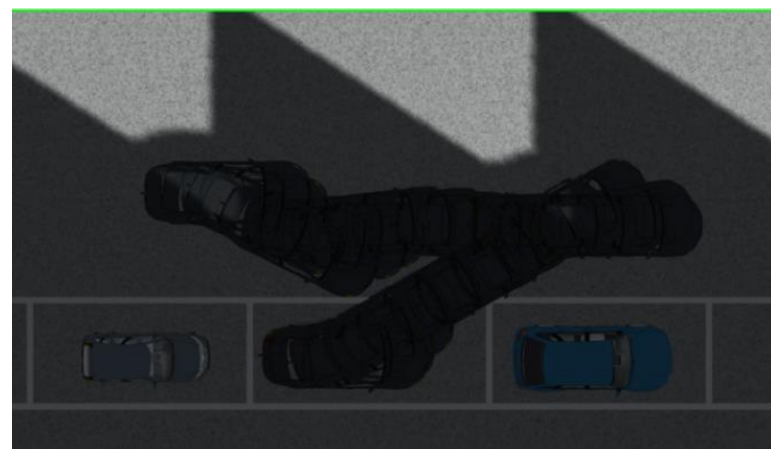

Figure 4. Simulation results of path planning

\subsection{Path tracking simulation}

Parking path will be automatically adjusted with different vehicle parameters, and in the process of looking for parking space, the linear movement of the vehicle is also controlled by the parking system. Therefore, the tracking control algorithm is used to verify the experimental results, which can objectively evaluate the response speed, tracking error and other indicators of the algorithm for path tracking. The tracing is shown in Figure 5.

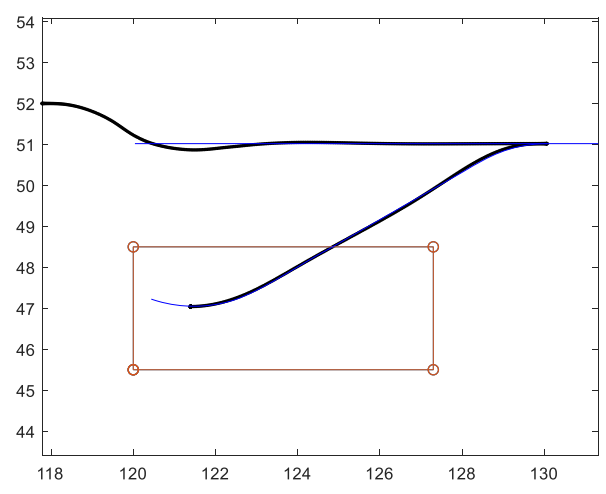

Figure 5. Path tracing

As can be seen from the figure, the automatic parking method proposed in this paper can well realize the tracking of the parking path when there is a large error in the initial pose, so that the vehicle can park into the parking space without collision, which meets the requirements of automatic parking and has good stability.

\section{Conclusion}

The most important function of automatic parking is to assist driving and adjust the parking track in time according to the change of parking environment. External environmental factors, such as light, weather, and choice of manual operation, can affect the automatic parking, and the deviation of the preferred position is too great, and may even lead to the failure of the parking. In order to overcome various defects, this paper uses the rapidly developing cloud service platform to clarify parking space information, adjust the position and attitude of parking vehicles before parking, and minimize the impact of environmental factors and human factors. At the same time, the sensor senses the obstacle information and the parking wireframe in the process of automatic parking, and uses the algorithm of cloud service platform to automatically plan the parking trajectory, which greatly improves the success rate and accuracy of parking.

\section{References}

1. Jiang Wuhua, Xin Xin, Chen Wuwei, Cui Weiwei. Multi-mode Parking Parking Recognition and Decision Planning of Automatic Parking System Based on Information Fusion [J/OL]. Chinese Journal of Mechanical Engineering :1-11[2021-03-13].

2. Li Chenxu,Jiang Haobin,Ma Shidian,Jiang Shaokang, Li Yue. Automatic Parking Path Planning and Tracking Control Research for Intelligent Vehicles[J]. Applied Sciences,2020,10(24).

3. Chai Runqi,Tsourdos Antonios,Savvaris Al,Chai Senchun,Xia Yuanqing,Chen C L Philip. Design and Implementation of Deep Neural Network-Based Control for Automatic Parking Maneuver Process.[J]. IEEE transactions on neural networks and learning systems,2020,PP.

4. Leopold Hrabovský,Hrabovský Leopold,Žáček Václav. Forces in the Lock-Off Device During the Rolling of the Brake Pulley Roller Over the Brake Ramp in the Built Automatic Parking House[J]. IOP Conference Series: Materials Science and Engineering, 2020,960(2).

5. Zhang Jiaxu, Wang Chen, Zhao Jian, Bu Chunyan. Path Planning and Tracking Control for Narrow Parallel Parking Space [J/OL]. Journal of Jilin University (Engineering and Technology Edition):17[2021-03-13].

6. VOROBIEVA $\mathrm{H}$, GLASER $\mathrm{S}$, MINOIUENACHE N, et al . Automatic parallel parking in tiny spots: path plan-ning and control $[\mathrm{J}]$.IEEE Transactions on Intelligent Transportation Systems, 2015,16( 1) : $396-410$ 